\title{
Visible Light Communication Survey
}

\author{
Eszter Udvary, Member, IEEE
}

\begin{abstract}
Communication applying visible light technology is a novel approach. Visible Light Communication (VLC) development is motivated by the increasing demand for wireless communication technologies. It has the potential to provide highspeed data communication with good security and improved energy efficiency. The rapid evolution of VLC was sustained by the LEDs performances. The Light-Emitting-Diode (LED) luminaires are capable of switching to the different light intensity at a fast rate. This function can be used for data transmission. This article focuses on the physical layer of the VLC links. It reviews the technology, the topology of the proposed connection, and the benefits of this approach. The main research trends are identified emphasizing state of the art in this area. It shows how VLC technology evolved and what are the performances achieved at this time. Various structures of the transmitter and receiver are studied, and different modulation schemes are investigated. Finally, numerous applications of VLC technology are presented.
\end{abstract}

Index Terms-Visible light communication, Optical-wireless communication, Free-space optical communication, Optical communication equipment, Modulation techniques, Machine-tomachine communications, Light emitting diodes, Lighting, Diode lasers

\section{INTRODUCTION}

$\mathrm{N}$ OWADAYS, a growing increase in the traffic carried by the telecommunication networks, including the wireless networks, can be observed [1]. The novel bandwidth-hungry applications increase the demand for broadband internet services, and further innovation, research, and development in the new emerging communication technologies are needed. The required capacity of wireless data transmission is expected to increase exponentially in the next years.

Radio frequency (RF) type communications are applied for wireless links, because of its maturity level and full acceptance. However, the radio frequency based wireless communications have some limitations. The reliability and the performances of the link are determined by the limited available spectrum and the increasing number of nodes. The main disadvantage is the limited bandwidth. There are also some scenarios where the RF caused interferences are critical, such as in aircraft, airports, or hospitals. So, novel wireless communication technologies are required.

Meanwhile, the development of the LEDs had massive growth. The revolution in the field of solid-state lighting leads to the replacement of fluorescent lamps by Light Emitting Diodes. Nowadays, LEDs are energy efficient, highly reliable,

Eszter Udvary is an Associate Professor at Budapest University of Technology and Economics, Budapest, Hungary (e-mail: udvary@hvt.bme.hu). and have a lifetime that exceeds by far the traditional light sources. So, LEDs are used in more and more lighting applications, because of the numerous advantages, and it is considered that LEDs will completely replace the traditional lighting sources [1] - [6]. On the other hand, LEDs can be used not only for lighting but also for communication, because the light intensity can be varied, and the switching speed is high enough.

Visible light communication is a new wireless communication technology which uses the white light not just for illumination purposes but also as a carrier for digital transmission. VLC uses the visible light (frequency range $=430-790 \mathrm{THz}$, wavelength range $=380-750 \mathrm{~nm}$ ) as a communication medium, which offers enormous bandwidths free of charge, this frequency range is safe to the human body, it does not disturb any sensitive electrical equipment, the allowed power is high, and it is not limited by any law, because of the applied non-licensed frequency range. As a visible light source can be used both for illumination and communication; therefore, it saves the extra power that is required in RF communication. The applied LEDs are energy efficient, small size, and cost-effective.

The basic concept is simple; the information modulates the intensity of the VLC transmitter. At the receiver side, a photosensitive element extracts the data from the detection of the fluctuation of the light intensity. The main advantage of VLC system is the application of the multifunctional device, which is used for lighting and data transmission same time. The communication link uses the existing LEDs lighting systems. With this approach, the implementation cost of the transmission link is significantly reduced.

Additionally, VLC link offers a considerable bandwidth available free of charge, enabling high data rate communications without any RF interference. VLC technology can provide low-cost, high-speed, optical-wireless data communication. VLC is a new technology, but the development is fast.

This paper aims at providing a survey to the physical layer of VLC technology. It presents the architecture of a VLC system, overviews the advantages and the disadvantages of the technology. This survey focuses on the applied modulation methods in the VLC systems. It identifies and discusses several top applications of VLC, pointing out the benefits of VLC usage. This article does not aim with the detailed VLC channel modeling and the standardization of VLC technology. The organization of this paper is as follows. Section II describes the architecture of VLC systems. Section III overviews the description of the potential applications of VLC. Section IV presents the state of the art of technology. Finally, section VI concludes the paper. 


\section{Visible Light COMMUNICATION LINK}

The VLC system consists of a VLC transmitter that modulates the white light produced by LEDs; a VLC receiver based on a photodiode that extracts the modulated signal from the light power, and the VLC wireless optical channel to connect the physically separated VLC transmitter and receiver. The simplified block diagram of a VLC system is presented in Fig 1.

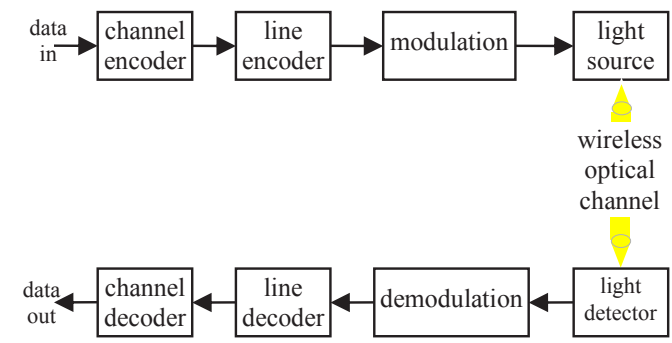

Fig.1. VLC link structure

\section{A. The transmitter side}

VLC transmitter transforms data into messages that can be sent over the free space optical medium by using visible light. The primary purposes that it is a multifunctional device; it emits light and transmits data at the same time. On the transmitter side, white light is generated by the LED, and the light is modulated by the information. The data transmission must not affect the primary illumination function of the device. From this point of view, the VLC transmitter must be met with the lighting requirements. So, the same optical power is used, or it is allowed for dimming. The dimming level that is selected for the modulation should be such that it is supported by the illuminating LEDs. On the other hand, the VLC transmitter must not induce any noticeable flickering. The modulation should be done in a way to avoid flickering.

Two types of white-light sources are used in solid-state lighting. Red-green-blue (RGB) emitter provides the white light applying three colors. The blue-LED on yellow-light emitting phosphorus layer provides white light by mixing blue and yellow lights. The VLC data transmitter can use both types, but RGB solution gets more modulation bandwidth. Contrary, the blue LED based device is more energy efficient and lower complex. Based on it, a blue LED with phosphorus is more popular in illumination systems. The RGB approach can be improved by applying a fourth color. RGBY model is supplemented by the yellow color, and therefore, there is not necessary to create complicated combinations of the fundamental wavelengths. As a result, the bitrate of the system is increased, and communication is possible on four independent channels. This fourth channel also improves area coverage [7].

The parameters of the VLC transmitter are mainly limited by the characteristics of the LEDs. The data rate depends on the switching abilities of the LEDs. The service area depends on the transmission power and the illumination angle. Currently, the industry produces LEDs that can offer switching frequencies of a few tens of megahertz. The modulation bandwidth is about $2.5 \mathrm{MHz}$ for the white component generated by a blue LED with yellow phosphorus. The switching speed of the blue LEDs is better, and higher data rates are enabled [8]. So, the modulation bandwidth can be increased by the filtering out of the yellow element in the receiver when only the blue part is detected. If this filtering eliminates the slow response of the yellow phosphorus, 14 $\mathrm{MHz}$ bandwidth can be achieved. Several other approaches are proposed to increase the bandwidth. Fully integrated LED driver design can provide high speed, low size, and economical power consumption solution. The $3 \mathrm{~dB}$ bandwidth of a VLC transmitter can be extended to $80 \mathrm{MHz}$ applying an integrated driver circuit with high pass transfer function [9].

To achieve higher VLC data rate, LASER (Light Amplification by Stimulated Emission of Radiation) diode transmitter has been proposed and demonstrated [10]. In this approach, the main challenge is the contemporary lighting and communication features. Nowadays, it is not applied in the lighting system.

Different types and forms of LED are applied in various environments. High power LEDs or LED arrays are used in illustrative in-door illumination purposes. Low-power devices are utilized in smart-phones and other mobile devices.

\section{B. Modulation techniques}

At the transmitter side, a dimming or biasing circuit with control function is necessary. Application of microcontrollers is a cost-effective solution for the encoder. The microcontroller can be replaced by a Field Programmable Gate Array (FPGA) in more complex applications. FPGA provides enhanced performances with the help of digital signal processing techniques. The encoder in the transmitter converts the data into a modulated message and manages the switching of the LEDs according to the binary data and the imposed data rate. So, the binary data are converted into an intensity modulated light beam. The visible light communication systems use intensity modulation and direct detection (IM/DD) approach. For IM/DD systems, the optical intensity must be real-valued and non-negative. As a result of the constraints of IM/DD, modulation schemes that are advantageous in radio frequency communications that may not be offered the same advantage in VLC. Typically, the light produced by the LEDs is current modulated with a baseband modulation signal. The baseband modulation schemes, like various type of On-Off Keying (OOK) modulation, Pulse Amplitude Modulation (PAM), and Pulse Position Modulation (PPM), are often applied [11]. Multicarrier modulation techniques as Orthogonal Frequency Differential Modulation (OFDM) can be used to increase the system capacity [12]. A unique modulation method, called Color Shift Keying (CSK) is specially designed for visible light communication to overcome the low data rate [13].

The baseband modulation schemes can be classified into pulse amplitude, pulse position, and pulse interval modulation depending upon the method information is encoded into the optical carrier. In On-Off Keying, the LEDs are turned off and 
on according to the bits in the stream; it is the same approach as the applied modulation in fiber optic systems. Typically, " 1 " bit is represented by the on state and " 0 " bit is represented by the off state. The LED is not turned completely off in the off state, because of the better modulation performance. The power requirement steadily decreases as the duty cycle decreases, but the bandwidth requirement increases. The implementation of OOK is easy and straightforward. The application of on-off keying modulation is limited by the slow time response of the yellow phosphor case of the blue emitter and yellow phosphor structure. It defines the modulation bandwidth. Typically, data rate up to $10 \mathrm{Mbps}$ can be realized using NRZ (Non-Return-to-Zero) OOK with white LED [14]. The data transmission rate can be improved with analog equalization, integrated driving circuits, and blue filtering techniques on the receiving side [9].

The low data rate OOK motivated researchers to develop new modulation techniques to achieve higher data modulation rates. In the case of Pulse Width Modulation (PWM), the width of the pulses varies according to dimming levels. The different dimming levels can be varied between $0 \%$ and $100 \%$ by applying high PWM frequency.

As the name suggests, the information in the Pulse Positioning Modulation scheme is encoded in the position of a pulse within a symbol. An L-PPM symbol consists of L time slots of equal duration. Within the symbol, all slots except the information bearing slot are empty. The position of this pulse carries information about the input bit sequence. The location of the pulse corresponds to the decimal value of the M-bit input data. So, a single pulse is presented in each symbol period; this scheme suffers from the problem of the low data rate. For smaller values of ' $L$ ', it is not efficient in terms of power and bandwidth usage. Multi-pulse PPM (MPPM) is a variant of PPM modulation schemes. It is more spectrally efficient because multiple pulses are transmitted in each symbol-time. A modified version of the PPM is the Expurgated PPM (EPPM) which was introduced to improve the performance of peak-power limited M-ary communication systems. The spectral efficiency of the MPPM and EPPM is less than 1, Multilevel EPPM (MEPPM) is proposed for the better spectral efficiency.

Color Shift Keying is a unique modulation method in VLC systems to enhance the data rate. This modulation scheme is designed to operate with RGB LEDs to provide higher order, spectrally efficient modulation. Three separate LEDs (Green, Blue, and Red) are utilized to produce the white light. Modulation in CSK is realized using the intensity of the three colors in an RGB LED source. Data are sent on the instantaneous color of the RGB triplet. CSK depends on the color space chromaticity diagram. The constant emitted light guarantees an absence of flicker at all frequencies. The constant luminous flux of the source leads to near constant current drive, which in turn implies a reduced inrush current when modulating data, strong signal isolation from the power line and a reduction in inductance caused by large switching currents. The bit rate is decided by the symbol rate and the number of color points on the constellation. That means the frequency response of the LEDs does not limit CSK bit rate. The main disadvantage of this approach, which Phosphorbased visible LEDs are more often used, and they are not suitable for CSK.

The VLC link has two main challenges: the limited bandwidth of the LEDs and the multipath propagation. The typical modulation bandwidth of LEDs is around couple tens of MHz. Complex modulation schemes such as phase shift keying (PSK), quadrature amplitude modulation (QAM) or OFDM modulations can be used to realize a higher data rate. The most popular and applicable choice in VLC systems is OFDM since it offers improved spectral efficiency than PSK, QAM and it has strong robustness against the intersymbol interference (ISI) airing from multipath propagation or limited system bandwidth.

High data rates exceeding $100 \mathrm{Mb} / \mathrm{s}$ are also attainable with multiple-subcarrier modulation techniques such as OFDM. With arrays of separately driven light sources and OFDM, data throughput of up to $1 \mathrm{Gbit} / \mathrm{s}$ was demonstrated, applying methods similar to radio frequency multiple-input and multiple-output (MIMO) approach. Multicarrier modulation schemes can be more efficient than the baseband modulation schemes. Table I. overviews the properties of the main baseband modulation schemes.

TABLE I

Properties OF BASEBAND MODULATION SCHEMES

\begin{tabular}{llll}
\hline \hline & Data rate & SNR & BER \\
\hline$O O K$ & medium & low & high \\
$P W M$ & very low & high & low \\
$P P M$ & low & high & medium \\
$M P P M$ & high & medium & high \\
$C S K$ & medium & medium & medium \\
MIMO OFDM & very high & high & low
\end{tabular}

The traditional OFDM signal widely applied to RF system is complex and bipolar. Due to IM/DD, the signaling for the VLC network must be a real and unipolar. Therefore, the traditional OFDM signal is modified to make them real-valued and unipolar. There are several variations of the unipolar OFDM that is proposed for VLC systems such as DC-biased optical OFDM (DCO-OFDM), asymmetrically clipped optical OFDM (ACO-OFDM), unipolar OFDM (U-OFDM), pulseamplitude modulated discrete multitone modulation (PAMDMT) and flip-OFDM. DCO-OFDM adds a DC-bias to the bipolar OFDM signal. The required DC-bias to satisfy nonnegativity is equal to the maximum negative amplitude of the OFDM signal. Negative signal clipping at the zero levels is applied to realize ACO-OFDM, which improves the power efficiency of the unipolar OFDM modulation format. Since only odd subcarrier is modulated, the ACO-OFDM has only the half the spectral efficiency of DCO-OFDM. However, there is no information loss when the signal is clipped, because of the anti-symmetry of the modulated signal. Pulse amplitude modulated discrete multitoned is similar to ACOOFDM [15], but the subcarriers are modulated by PAM. In a PAM-DMT system, there is no DC bias. All of the subcarriers are modulated, but the modulation uses only the imaginary 
part of the subcarrier [16]. Thus the spectral efficiency is the same as ACO-OFDM. Although it has limited spectral efficiency, it is more power efficient than DCO-OFDM, because it also has an anti-symmetry (Hermitian symmetry). Unipolar OFDM (U-OFDM) is almost the same concept named Flip-OFDM. In this case, the negative and the positive part of the real bipolar OFDM signal are extracted. Hence, Hermitian-symmetry is preserved. The polarity of the harmful components of the symbol is inverted before the transmission of both positive and negative elements in a consecutive OFDM symbol. Table II summarizes the properties of the OFDM schemes.

TABLE II

PROPERTIES OF OFDM SCHEMES

\begin{tabular}{llll}
\hline \hline & $\begin{array}{l}\text { power } \\
\text { efficiency }\end{array}$ & $\begin{array}{l}\text { spectral } \\
\text { efficiency }\end{array}$ & $\begin{array}{l}\text { Hermitian } \\
\text { symmetry }\end{array}$ \\
\hline DCO OFDM & good & good & anti-symmetry \\
ACO-OFDM & improved & half & anti-symmetry \\
$P A M-D M T$ & improved & half & anti-symmetry \\
flip-OFDM & & &
\end{tabular}

\section{The receiver side}

The VLC receiver extracts the data from the modulated light beam. The optical lens at the receiver collects and concentrates the incoming light to a photo-detecting element. Imaging and non-imaging receivers may be used. The detection bandwidth is typically higher than the limit of the transmitter and channel dispersion. In mobile devices, like smartphones, tablets, low-cost photodiode, or optical sensor is applied. At the output of the IM/DD link, the light power is converted to current by the photodiode. The produced current signal is proportional to the intensity of the incident wave and depends on the photodiodes spectral sensitivity.

So, the photodiode transforms the light into an electrical signal that will be demodulated and decoded by the embedded decoder module. Depending on the required performances and the cost constraints, the decoder can be a microcontroller or an FPGA. The VLC receivers are based on typically a reverse biased photodiode operating in a photoconductive mode which has high bandwidth and offers the possibility of high-speed communications. At the electrical output of the receiver, significant interference can be observed, because of the other artificial or natural light sources. An optical filter can enhance the performances of the VLC receiver. The optical filter decreases the unwanted spectrum components. Moreover, in high-speed applications using white LEDs, the optical filter allows only the passage of narrowband radiation, corresponding to the blue color.

The effect of the interferences can also be reduced by narrowing the receiver field of view (FOV), but it influences the service area. If wider FOV is applied, a more extensive service area can be supported. But more noise is captured, and the Signal to Noise Ratio (SNR) is degraded. Indoor shortrange applications require increased mobility, and narrow FOV approach is not useful in these scenarios. In case of outdoor long range applications, the narrow FOV is a practical solution, because the long-range link induces small angles, anyhow. A narrow FOV improves the robustness of the VLC system again, the noise due to daylight or from other VLC transmitters [17]. The FOV of the whole receiver is determined by the FOV of the optical focusing system, which concentrates the light on the photodetector by using a lens.

Similarly, the performances of the system can be enhanced by increasing the area of the photodetector. However, if the area of the photodetector is large, its capacitance is also increased. The capacitance and the load resistor limit the available bandwidth. The applied photodetectors area represents a trade-off between SNR and bandwidth. The generated photocurrent is low, and a transimpedance circuit is used to transform the small current into voltage. The transimpedance solution offers a trade-off between the gainbandwidth product and noise.

The output voltage of the transimpedance circuit is amplified and filtered to remove high and low-frequency noises, and the DC component. Finally, the data processing unit decodes the information from the reconstructed signal obtaining the binary message.

\section{The VLC channel}

The transmitter and the receiver are interconnected through the free space optical communication channel. Practically, the visible light is an electromagnetic wave; as any electromagnetic radiation, the intensity of the visible light decreases with the square root of the distance as it passes through the channel. This paper does not aim with the detailed VLC channel modeling; just a short overview is included.

An optical wave propagating in an unguided medium has to go through many constituents, which are different types in different environments. The molecules and aerosols are the main absorbers in the atmosphere. On the other hand, water molecules, chlorophyll, colored dissolved organic and suspended particulate matters; dissolved salts affect the propagation in the underwater medium. These constituents cause the optical wave to get scattered and absorbed, which in turn results in the degradation and attenuation of the received optical signal.

The VLC transmission channel is affected by numerous sources of optical noise. In the daytime, the most critical noise source is the sun. Other sources of noise are represented by other VLC transmitters or any source of light with or without data transmission capabilities. In outdoor environments, the weather causes more problems for VLC applications. The rain, snow, or dense fog includes water particles. It causes scattering of the light containing the data and affects the performance of the VLC link. The noise sources and the low signals significantly degrade the SNR in VLC, especially at a long distance link. There are several possibilities for enhancing the SNR at the receiver, like optical filtering, the adequate design of the optical system, or adaptive electrical gain and filtering.

The turbulence is the random refractive index in time and space due to the circulation of air or water, which cause fluctuation in the received signal. The turbulence is developed mainly by the variations in the temperature, pressure, and humidity. The applied formulations for turbulence power 
spectra are various in different environments such as atmosphere, space, and underwater. The average intensity and the scintillation index are often calculated based on the Rytov method and the extended Huygens-Fresnel principle. Effects of different optical beam profiles, the transmitter and the receiver aperture averaging to reduce the turbulence degradation can also be taken into account [18].

In VLC link the multipath propagation is experienced mainly at indoor short transmitter-receiver distances [19] and unique scenarios as underwater and vehicular applications. Besides the LOS component, there are a large number of reflections among ceiling, walls, and floor as well as any other objects within the environment. The rays of light hit the other walls and are reflected towards the receiver. The receiver can only detect the rays entering its field of view. The detailed channel model of visible light communication system takes into account the position, size, and shape of the obstacle in the illumination area of the light source [20]. Same results can be observed within the marine environment. The reflection characteristics of the sea surface and sea bottom, as well as the presence of human and human-made objects, determine the communication [21]. In the vehicular applications, the primary reflectors are the other cars which are located in the next lanes [22].

\section{Purposes Of The Visible Light COMMUNiCATION}

\section{A. VLC advantages}

VLC can be considered to be the next generation of wireless communications, because of the unique characteristics and advantages. VLC can solve some of the problems of RF communication. The benefit of VLC comes from the benefits of the visible light. High bandwidth can be used for free of charge, which allows high data rates, unlicensed spectrum, and safety for the human body and high-precision electronic equipment. From the point of security, VLC is also better than radio frequency communication. Besides these advantages, VLC is a low-cost technology, and the implementation is straightforward as it uses the same infrastructure than a lighting system.

1) High bandwidth

The RF communications have up to $300 \mathrm{GHz}$ available bandwidth, which is used for different types of applications, and consequently, the networks are often saturated. Both radio- and television broadcasting, GSM (Global System for Mobile Communications, formerly Groupe Spécial Mobile), satellite communications, military applications, etc. use the same RF frequency range. The extension of the bandwidth is costly, and sometimes it is not possible, at all. On the other hand, the complexity of the higher frequency equipment increases, and the devices are expensive. Most of the frequency ranges are licensed; the available bandwidth in unlicensed frequency ranges is limited.

VLC uses the visible light spectrum, which is between 380 and $780 \mathrm{THz}$; it adds $400 \mathrm{THz}$ available bandwidth for the wireless communications. The VLC can use worldwide an unregulated and almost unlimited bandwidth offering multi$\mathrm{Gb} / \mathrm{s}$ data rates.
2) Unrestricted technology

In social environments, like hospitals or aircraft the radio frequency communication is restricted. The reason that RF communication may cause malfunctions of the high precision electronic equipment. Light communication does not affect any RF signal or equipment, because of the elimination of electromagnetic interference. So, VLC is safe for these places.

\section{3) Security}

There is no wireless communication method without risking of eavesdropping. Only quantum communication wireless channel can discover the presence of an eavesdropper, based on the photon statistic [23]. But, compared with radio frequency wireless communication approaches, the visible optical-wireless link provides higher security against eavesdropping, because the light cannot penetrate through walls. The main security limitation of indoor VLC channel is the point when the wall is open. The common weakness is the window. When the eavesdropper is in a LOS position, it and can receive the signal via the window [24]. The VLC transmitters are located near the window are more endangered. But, far VLC transmitters can also be affected via reflections from the walls and indoor equipment. On the other hand, theoretical eavesdropping of VLC based communications is a possibility through keyholes and door gaps. So, careful and adequate planning of the system is required in sensitive areas. However, VLC technique provides higher security than other radio frequency wireless links. Based on it, the VLC link is suitable in military applications or areas of high security.

4) Low implementation cost

There are three main reasons for the lower price of VLC compared with other wireless technologies. First, RF systems use a regulated band. Contrary, the visible light is in an unlicensed region of the electromagnetic spectrum. The implementation cost is significantly reduced because there is no cost for a license. Secondly, VLC will rely on existing infrastructures that are already accepted and widespread across the world. So, VLC is simple, without requiring complex modifications on the existing infrastructure. It also decreases the implementation cost. The third aspect is its reduced complexity. VLC primarily uses LED transmitters and photodiode receivers; these components are inexpensive.

5) Green wireless communication technology

The natural resource consumption and climate deteriorations are increasing as the Earth's population are growing, and human society is developing. Greenhouse gas emissions have reached alarming levels; it produces significate climate changes that affect the whole ecosystem [25]. Natural resource consumption and pollution can be significantly reduced by decreasing energy consumption. A significant percentage of energy consumption comes from artificial lighting, commonly provided by electric lights. Worldwide, approximately $20 \%$ of electricity is used for illumination, while electricity represents about $15 \%$ of the total energy produced [25].

VLC is a green wireless communication technology because it does not use additional power for communication. The same infrastructure and light are used for illuminating are also used 
for data transmission. Another essential advantage of VLC is the utilization of LEDs which provide substantial energy savings, reducing the $\mathrm{CO}_{2}$ emissions.

6) Safe for human health

The application of visible light for data transmission is entirely safe for human health. The RF adverse effect on human health is not adequately demonstrated. But, RF electromagnetic waves are currently classified as a possible cause of cancer in humans by the World Health Organization. The infra-red light (IR) is also used for wireless communications, but it has a heating effect on the incident surface. So, high power IR light can cause irreversible thermal damage of the cornea, making it harmful for the human eye [26].

\section{B. VLC Challenges}

As a conclusion of the previous sub-session, VLC is a technology that has plenty of essential advantages. Naturally, VLC has also some drawbacks. Most of the disadvantages are due to the early stage of the VLC technology. It could be overtaken as the technology is fully developed. The other ones are due to the usage of the light and its characteristics. Completely mitigate them is difficult, but their effects could be reduced, or the communication could be adapted to the situations.

\section{1) The Line of Sight condition}

VLC mostly requires line-of-sight (LoS) transmission; otherwise, the received light signal may be very weak. In a general aspect, LoS maximizes power efficiency and minimizes multipath distortion. Additionally, the interferences from other receivers are limited, and communication security is enhanced. However, Non-LoS communications are more reliable, flexible, and robust. The necessary LoS condition hurts mobility and, in some areas, it represents VLC's most significant disadvantage because an object interposed between the transmitter and receiver can block the communication unless an alternate route is available [27]. In multi-hop communications and retransmissions, the data can reach users that are located outside the transmitter's LoS but are in the serving area of another transceiver [28]. VLC system can be combined with RF links [29] when VLC cannot address a node; RF serves it.

2) Limited transmission range

VLC cannot reach the same transmission range than RF communications [30]. The VLC transmission range can be increased by optimizing the transmitter and receiver parameters, but it is still significantly shorter than the RF communication range. On the transmitter's side, the communication range can be increased by increasing the transmission power or by using a more directive light beam. So, the visible LASER diode can reach a larger distance than LED, but with this device, we lost the main advantage of the system, the LASER source cannot be used as a multifunctional transmitter. On the receiver's hand, the range can be improved by using different techniques for SNR enhancement, such as narrow FoV receiver, an optical lens or different filtering techniques. Additionally, multi-hop networking can also increase the communication range of the VLC network.

3) Susceptibility to interferences

VLC is affected by the light from other incandescent or fluorescent light sources [31]. Typically, these light sources produce low-frequency noise which can be removed with a high pass filter. In outdoor applications, the sunlight causes strong perturbation. The sun produces unmodulated light, which creates a strong DC electrical component at the output of the receiver. It can be removed with capacitive DC filter. However, high-intensity optical noise can saturate the receiver and block the communication.

The degrading effect of other light sources can be reduced by optimizing the receiver. Optical filters, reduced receiver FOV, and electrical filter can eliminate the unwanted frequency components. Even if the mentioned technique mitigates the effect of the interferences, high levels of noise still affect the communication performances.

\section{APPLiCATIONS}

Essential features of VLC include high bandwidth, no health hazard, low power consumption, and non-licensed channels made it attractive for practical use. By taking benefit of the mentioned advantages, VLC technology can support numerous applications. VLC seems to be the only choice in some of the application. But it can be a complementary solution for RF communications, improving the overall performances in several other scenarios. The applications can be grouped into high speed and low-speed communication types. In the high-speed applications, the primary goal of the development the higher bandwidth and the better utilization of the available bandwidth to realize higher and higher transmission bitrate.

Another classification is based on the application area; indoor and outdoor applications can be defined.

\section{A. $\mathrm{Li}-\mathrm{Fi}$}

One of the essential high-speed application envisioned for VLC is providing of Light-Fidelity or "optical Wi-Fi." In 2011, Prof. Harald Haas was the first one used the term Light Fidelity (Li-Fi) [32]. The considerable potential and the fast evolution of the Li-Fi technology contributed to the foundation of the Li-Fi Consortium in the same year [33]. LiFi is a high speed, bi-directional, fully connected, visible light wireless communication system and is analogous to wellknown Wi-Fi. The system can enable high-speed internet connections from the ceiling lamp, because of the large available bandwidth. The limited VLC link length does not limit the scenario, because the required distance is a few meters, equivalent to the distance between the ceiling and ground in an office. This technology based on Li-Fi routers can offer multi $\mathrm{Gb} / \mathrm{s}$ connections. The data, which comes from the internet, is transformed into a driving signal of the light source. According to the data, the light source will switch on and off at frequencies unperceivable by the human eye. The receiver converts the light signal into an electrical signal and next into numerical data which will be delivered to the mobile terminal. 
Fig. 2. shows the basic idea of the Li-fi concept.

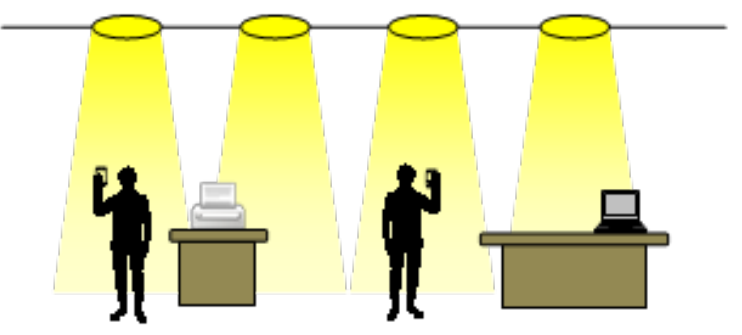

Fig.2. Li-Fi idea

There are several approaches to realize the uplink in Li-Fi system. Visible light sources could provide both up- and downlink. But the realization is not useful in all cases, because of the lack of installed VLC infrastructure for the upload. On the other hand, mainly downlink connection pushes the huge bandwidth advantage of VLC link. Typically uplink is performed using an infrared link [34], or the already existing Wi-Fi uplink [35] in integrated Wi-Fi and Li-Fi service.

The transmission of the VLC signal does not affect the already applied RF-based communications system. So the two alternative wireless technologies could be used together. It means that the two technologies can cooperate; the VLC link can take some of the load from the already full RF spectrum. One useful approach, then the VLC technology, offers high data rate downstream from the router to the mobile terminal. However, the upstream is provided by radio frequency communication from the mobile terminal to the router [35].

\section{B. Optical-wireless communication in RF restricted areas}

In areas where RF communications are limited, VLC technology can be safely used to provide wireless connections. It can be a safe alternative to radio frequency communications in hazardous environments, such as mines and petrochemical plants. It is also useful in applications where traditional WLAN communication may interfere with specialized equipment, like hospitals and aircraft passenger cabins' inflight entertainment systems

A most studied example is the hospital environment, where electromagnetic wave sensitive areas should connect to the network. The utilization of RF communications in hospitals and health care units are restricted, especially in operating theatres and around magnetic resonance scanners. In these places, the information exchange is possible by using a VLC system, because VLC does not interfere with radio waves of the other equipment and machines.

Aviation is also a restricted area for RF communications. Radio is undesirable in passenger compartments of aircraft. LEDs are already used for illumination and can also be used instead of wires to provide media services to passengers. This approach reduces aircraft construction costs and weight. VLC can also be used in hazardous environments where there is a risk of explosions. In these areas, RF communications are restricted due to the risk. VLC can be successfully used in these areas, like mines, oil rigs, chemical plants, etc. Additionally, the communication capability is a complement to the already existing LED-based lighting systems.
On the other aspect, VLC uses light sources, which is typically not LASER, but the system must follow the safety regulations and limits. Accessible Emission Limit (AEL) is the maximum affordable emission level permitted within a particular LASER hazard class. Maximum Permissible Exposure (MPE) is the level of LASER radiation to which an unprotected person may be exposed without adverse biological changes in the eye or skin. The nominal hazard zone (NHZ) can be determined. A complex calculation may have to be performed to assess AEL, MPE, and NHZ values for certain LASERs and specific conditions. This calculation is defined in standards and regulations. However, VLC links typically apply LED-based lighting system, which does not exceed the limits, determined for LASER based system.

\section{Indoor localization}

In the indoor environment, VLC based localization is very convenient since the classical GPS is not able to work inside buildings [36]. The omnipresence of LEDs for illumination provides unique opportunities for indoor localization [37]. Visible light can be applied as an ID system in unusual places such as buildings and subways. The visible light ID system identifies the location, typically the room and the building. Similarly, the visible light ID system can be introduced in subways, hospitals, and airports. The system can be extended, when the ID provides the coordinates of the transmitter.

Signals transmitted by the LEDs can be used to determine the position of a person or object within a room with high precision. The requirements of such applications are different from high-speed VLC systems. One of the commonly employed methods for VLC based localization is optical tracking and imaging while the other is based on trilateration/triangulation. However, in all two purposes, it is critical that the user device can recover signals from each luminaire separately.

Almost all location determining methods are based on the apriori knowledge of the position of communicating LED devices playing a role in the localization. Fig. 3. presents the VLC based indoor localization concept. For this, we have to know which transmitter's signal has been detected. So, multiplexing methods are required for the VLC system. Mainly three types of channel access technique can be implemented in VLC systems; time, frequency, and code division multiple access [38].

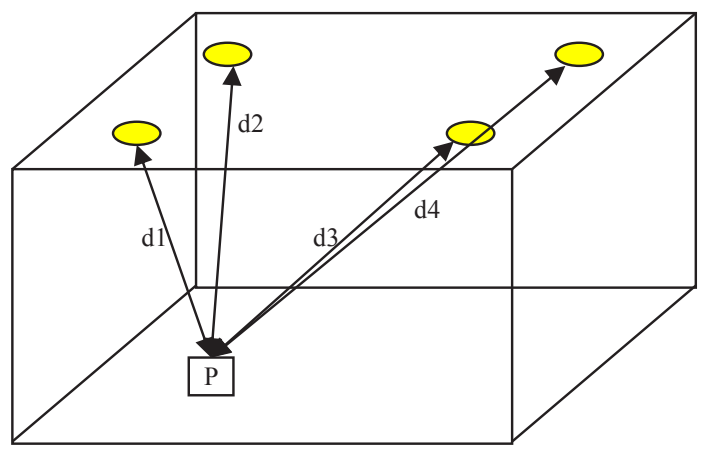

Fig.3. VLC based indoor localization 
On the other hand, $\overline{\mathrm{L}} \overline{\mathrm{L}} \overline{\mathrm{C}}^{-}$can provide very efficient indoor localization. Centimeter accuracy can be approached by determining the received signal strength or the time of flight and by using the triangulation technique.

\section{Location-aware applications}

Over the years, smart advertising has become an essential marketing tool for brands to communicate with their potential customers in a place where a large number of people live. VLC could also be used to create smart areas by providing geo-localized information. This way, the location-aware information can be delivered to users' smartphones or tablets by using the indoor light. Information Displaying Signboards are often made from an array of LEDs; these signboards are installed to distribute information in airports, bus stops, and other places where the broadcasting of information is necessary. The signboard can also transmit data; this type of signboard can be applied for indications in several locations such as airports, museums, and hospitals.

The indoor public areas can be equipped with accurate positioning and localization systems based on VLC technology. Several applications may use this indoor positioning service. In theaters or cinemas, guide audience can help the visitors to their seats.

The localization information can trigger a particular audio or video guide script in a museum. The museum could use LiFi to provide information about exhibitions [39].

Similar to museums, retail markets could provide product information, coupon codes, and other personalized shopping experiences. It can help shoppers to find discounted items in a store or supermarket. Supermarkets are ideal advertising spaces since hundreds of consumers visit them every day and are receptive to the advertising that is offered to them. The process consists of delimiting the zones in which the owner of the supermarket wishes to deploy his advertising campaign through the positions of the LEDs suspended from the ceiling. Consumers may prefer Li-Fi in this case to Bluetooth or Wi-Fi since it allows them to remain anonymous by only receiving data.

\section{E. Transportation}

The most promising outdoor application of VLC technology is the intelligent transportation system applying the vehicle lights and the existing traffic light infrastructure. The LED headlights and taillights are commercially available in cars. The street lamps and traffic signals are also moving to LED technology. So, VLC based vehicle-to-vehicle and infrastructure-to-vehicle communications are achievable. It can manage road safety and traffic management. By using an optical wireless connection, safety messages can be transmitted from one vehicle to another one; and also from the traffic infrastructure to the approaching vehicles. Cars share data concerning their state, like velocity, location, acceleration, braking action, or their mechanical state, etc. (Fig.4.). Based on it several intelligent transport system services and applications can be realized including cooperative forward collision warning, emergency electronic brake lights, lane change warning, pre-crash sensing, stop sign movement assistant, traffic signal violation warning left turn assistant, etc. On the other hand, cars can distribute and disperse traffic information, like the place of an accident in the system. This approach can improve the safety and efficiency of the transportation system. Based on the distributed information location services and optimized alternative routes can be offered [40].

In crowded cities or on highways we have to calculate with high traffic density scenario. In this case, the ability of radio frequency communications to support intelligent vehicular communication has limited reliability because of mutual interferences. VLC is a line of sight technology, and it is not affected by interferences. So, visible light transmission can successfully support communications in high traffic density.

It is an outdoor application. So, the system must be robust to perturbations noises from the sunlight or the artificial light. The solution has also to be cost effective to make sure that high market penetration is possible.

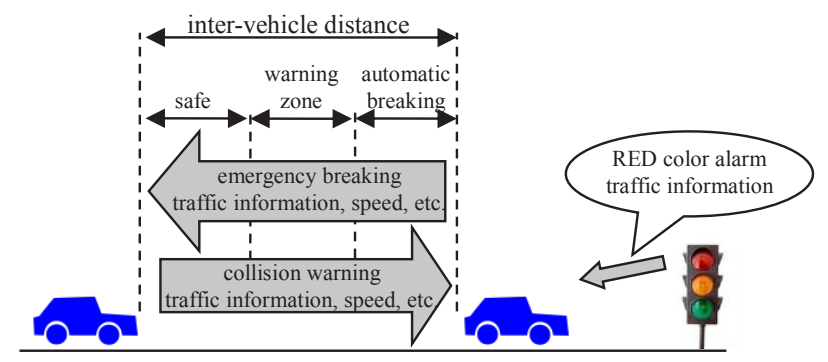

Fig.4. Intelligent transport system based on the VLC technology

\section{F. Underwater communications}

Data transmission in an unguided water environment through wireless carriers is called as underwater wireless communications. Radio-frequency wave, acoustic wave, and optical wave can be applied in this system. RF waves do not propagate well in seawater; it is not able to provide underwater communications. The bottlenecks of the acoustic transmission are the low bandwidth, high latency, and complex energy-consuming acoustic transceivers. VLC can be effectively used in this environment. In comparison to RF, underwater optical wireless communication can provide a much higher transmission bandwidth and much higher data rate. In this case, VLC can provide short-range (some meters) communications which can enable divers, underwater robots, and undersea sensors to communicate with each other or with the base station or buoy on the surface (Fig.5).

VLC technology may achieve a data rate on the order of Gbps over moderate distances of tens of meters in seawater. The transmission speed of light in water is much higher than the acoustic wave; the VLC link provides low link latency. The high transmission data rate and the low latency guarantee the realization of real-time applications like underwater video transmission. Underwater optical-wireless also has higher communication security over the acoustic and RF methods. Eavesdropping is more difficult in LoS configuration, which is implemented in VLC systems, rather than the diffused broadcasting scenario like acoustic and RF wave 
communication. Finally, the optical-wireless link is much more energy efficient and cost-effective than its acoustic and RF competitors. Acoustic and RF transceivers are large, expensive, and highly energy consuming. Underwater VLC transceivers are relatively small and low-cost implementing LASER diodes or LEDs and photodiodes. [41]

Besides the advantages of marine VLC technology, there are some disadvantages or at least challenges of the approach.

The blue and green spectrum components are used for underwater communication, because of the minimum attenuation value. However, the optical signal is still degraded by the absorption, and the scattering caused multipath propagation due to the inevitable photon interactions with the water molecules and other particulate matters in the water. In an underwater environment, chlorophyll, similar issues, and colored dissolved organic materials are capable of absorbing the blue and red lights [42]. Additionally, its increase the turbidity of the water and thus shrink the propagation distance of the light. Moreover, the concentration of colored dissolved organic material will also change with ocean depth variations, and consequently, the corresponding light attenuation coefficients also vary with the water depth.

VLC communication in the marine environment is a unique situation. In this application, there is no already installed lighting infrastructure; the VLC communication link is established for the dedicate data transmission. So, blue or green LASER can be implemented instead of an LED light source. The LASER source has a narrow divergence feature, which increases the safety and the power budget. However, LASERs with a tight divergence angle require precise pointing between transmitter and receiver, an exact alignment condition is needed. This requirement will limit the performance of the system in turbulent water environments and can become a critical problem when the transmitter and the receiver are nonstationary nodes, such as an autonomous vehicle. Underwater optical links will be temporarily disconnected due to misalignment of optical transceivers. It can take place frequently, as the underwater environment is turbulent, especially in the vertical buoy-based surface-to-bottom applications. Random movements of sea surface will cause serious connectivity loss problem. Use of a LED light source with more extensive divergence may solve this problem. However, the wider divergence of the light ray comes from the LED source limits the link length.

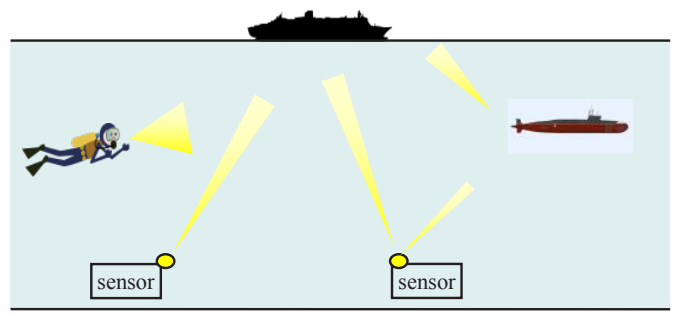

Fig.5. Underwater VLC transmission
Implementation of the systems requires reliable underwater devices. In the marine environment, the flow, the pressure, the temperature, and the salinity of seawater strongly impact the performance and lifetime of the underwater devices. The reliability of device batteries and efficiency of device power consumption are critical because solar energy cannot be exploited in the undersea environment.

\section{CONCLUSION}

Visible light communication has the potential to provide high-speed data communication with good security and improved energy efficiency. VLC development is motivated by the increasing demand for wireless communication technologies. The LEDs performances sustained the rapid evolution of VLC. VLC can be successfully commercialized in coming years because the interest increases from the research community, industries, and standardization.

This paper is an overview of literature covering features and applications of visible light communication. It first introduces the motivation for VLC technology development. The main advantages and disadvantages of this technology are overviewed; the challenges are presented. The main applications of VLC technology were identified.

The different modulation techniques are discussed, the optimal modulation scheme should be able to provide dimming support and minimize flickering effect while maintaining higher spectral efficiency. This part of the paper includes a review of major modulation techniques (OOK, PPM, OFDM, and CSK).

The last sections provide an overview of communication applications. High data rate indoor communication is one of the main application domains for VLC technology. It can be used for fast internet connection or a fast data broadcast over a short communication range. The scientific community has made significant efforts in this research area, which allowed VLC to obtain impressive results.

On the other hand, several outdoor applications serving more extended range are also developed by the research community. Both underwater communication and automotive area require special requirements. The main challenges in these applications are the achievement of tens of meters communication and increasing the robustness to noise. The third main area is the low bitrate approaches, mainly sensor and identification applications.

\section{REFERENCES}

[1] Murat Uysal, Carlo Capsoni, Zabih Ghassemlooy, Anthony Boucouvalas, Eszter Udvary: Optical Wireless Communications, An Emerging Technology, ISBN 978-3-319-30201-0, Springer International Publishing, 2016

[2] Zabih, Ghassemlooy; Luis, Nero Alves; Stanislav, Zvánovec; Mohammad-Ali, Khalighi: Visible Light Communications: Theory and Applications, New York, United States, Boca Raton, CRC Press, 2017

[3] Z Ghassemlooy: Visible Light Communications - A Review, MMTC Communications - Frontiers, Vol. 12, No. 3, May 2017 pp 6-13

[4] Steigerwald, D.A; Bhat, J.C.; Collins, D.; Fletcher, Robert M.; Holcomb, M.O.; Ludowise, M.J.; Martin, P.S.; Rudaz, S.L., "Illumination with solid state lighting technology," Selected Topics in Quantum Electronics, IEEE Journal of, vol.8, no.2, pp.310,320, Mar/Apr 2002.

[5] Azevedo, I.L et al.: "The Transition to Solid-State Lighting," Proceedings of the IEEE, vol.97, no.3, pp.481,510, March 2009. 
[6] Hanzo, L.; Haas, H.; Imre, S.; O'Brien, D.; Rupp, M.; Gyongyosi, L. "Wireless Myths, Realities, and Futures: From 3G/4G to Optical and Quantum Wireless," Proceedings of the IEEE, vol.100, no. Special Centennial Issue, pp.1853,1888, May 13, 2012.

[7] Yiguang Wang; Li Tao; Xingxing Huang; Jianyang Shi; Nan Chi: 8Gb/s RGBY LED-Based WDM VLC System Employing High-Order CAP Modulation and Hybrid Post Equalizer, IEEE Photonics Journal, Volume 7, Number 6, December 2015

[8] Hoa Le Minh; Dominic O'Brien; Grahame Faulkner; Lubin Zeng; Kyungwoo Lee; Daekwang Jung; YunJe Oh; Eun Tae Won "100-Mb/s NRZ Visible Light Communications Using a Postequalized White LED," IEEE Photonics Technology Letters, 2009, Volume: 21, Issue: 15 Pages: $1063-1065$

[9] Dong Yan1, Xurui Mao2, Sheng Xie3, Jia Cong1, Hongda Chen: Design fully integrated driver circuit for phosphorescent white Light-Emitting-Diode highspeed real-time wireless communication, accepted for publication, DoI: 10.1109/JPHOT.2019.2904607, IEEE Photonics Journal

[10] Chien-Hung Yeh1 et al.: $1250 \mathrm{Mbit} / \mathrm{s}$ OOK Wireless White-Light VLC Transmission Based on Phosphor Laser Diode, IEEE Photonic journal, 2019

[11] Visible Light Communications: Theory and Applications, Chapter 5: Tamas, Cseh, Sujan, Rajbhandari, Gabor, Fekete, Eszter, Udvary, Modulation Schemes, New York, United States, CRC Press, 2017

[12] Tsonev, D.; Hyunchae Chun; Rajbhandari, S.; McKendry, J.J.D.; Video, S.; Gu, E.; Haji, M.; Watson, S.; Kelly, AE.; Faulkner, G.; Dawson, M.D.; Haas, H.; O'Brien, D., "A 3-Gb/s Single-LED OFDMBased Wireless VLC Link Using a Gallium Nitride uLED," Photonics Technology Letters, IEEE , vol.26, no.7, pp.637,640, April1, 2014.

[13] Carlos E. Mejia; Costas N. Georghiades: Coding for Visible Light Communication Using Color-Shift-Keying Constellations, IEEE Transactions on Communications, 2019

[14] Haiqi Zhang et al.: Gb/s Real-Time Visible Light Communication System Based on White LEDs Using T-Bridge Cascaded PreEqualization Circuit, IEEE Photonics Journal, 2018, Vol.10 , Issue: 2

[15] Haoxu Li; Jin Wang; Xiaofeng Zhang; Rangzhong Wu: Indoor visible light positioning combined with ellipse-based ACO-OFDM, IET Communications 2018, Volume: 12, Issue: 17, Page s: $2181-2187$

[16] Tian Zhang; Yue Zou; Jianing Sun; Shuang Qiao: Design of PAMDMT-Based Hybrid Optical OFDM for Visible Light Communications, IEEE Wireless Communications Letters, 2019, Volume: 8, Issue: 1 Page: $265-268$

[17] Cuiwei He; Thomas Q. Wang; Jean Armstrong: Performance of Optical Receivers Using Photodetectors With Different Fields of View in a MIMO ACO-OFDM System, Journal of Lightwave Technology,

[18] 2015, Volume: 33, Issue: 23, Page s: 4957 - 4967

[19] Xi Nan; Ping Wang; Lixin Guo; Li Huang; Zhongyu Liu: A Novel VLC Channel Model Based on Beam Steering Considering the Impact of Obstacle, IEEE Communications Letters ( Early Access ), 2019

[20] Kwonhyung Lee; Hyuncheol Park; John R. Barry: Indoor Channel Characteristics for Visible Light Communications, IEEE Communications Letters, 2011, Volume: 15, Issue: 2, Pages: 217 - 219

[21] Farshad Miramirkhani, Murat Uysal: Visible Light Communication Channel Modeling for Underwater Environments With Blocking and Shadowing, IEEE Access, February 14, 2018.

[22] Hasan Farahneh, Fatima Hussain, Xavier N Fernando: Performance analysis of adaptive OFDM modulation scheme in VLC vehicular communication network in a realistic noise environment, EURASIP Journal on Wireless Communications and Networking 2018

[23] Agoston Schranz, Eszter Udvary: Quantum Bit Error Rate Analysis of the Polarization-based BB84 Protocol in the Presence of Channel Errors, Photoptics 2019

[24] Ignacio Marin-Garcia; Victor Guerra; Patricia Chavez-Burbano; Jose Rabadan; Rafael Perez-Jimenez: Evaluating the risk of eavesdropping a visible light communication channel, IET Optoelectronics Year: 2018, Volume: 12, Issue: 6

[25] Walsh, J., Donald Wuebbles: Our Changing Climate, Climate Change Impacts in the United States: The Third National Climate Assessment, U.S. Global Change Research Program, Dor: 10.7930/J0KW5CXT. chapter 2, pages 19-67

[26] James C. Lin: Current Activities on Exposure Limits for Humans in the Radio-Frequency Region, IEEE Antennas and Propagation Magazine, 2014, Volume: 56, Issue: 6, Pages: 256 - 258
[27] Giulio Cossu; Raffaele Corsini; Ernesto Ciaramella: High-Speed Bidirectional Optical Wireless System in Non-Directed Line-of-Sight Configuration, Journal of Lightwave Technology, 2014, Volume: 32, Issue: 10, Page s: 2035 - 2040

[28] Xun Guan, Qing Yang, Taotao Wang, Calvin Chun-Kit Chan: Phase-Aligned Physical-Layer Network Coding in Visible Light Communications, accepted paper, IEEE Photonics Journal DOI: $10.1109 /$ JPHOT.2019.2904954.

[29] Sihua Shao; Abdallah Khreishah; Moussa Ayyash; Michael B. Rahaim; Hany Elgala; Volker Jungnickel; Dominic Schulz; Thomas D. C. Little; Jonas Hilt; Ronald Freund: Design and analysis of a visible-lightcommunication enhanced WiFi system, IEEE/OSA Journal of Optical Communications and Networking, Year: 2015, Volume: 7, Issue: 10, Pages: 960 - 973

[30] Chen Gong; Shangbin Li; Qian Gao; Zhengyuan Xu, Power and Rate Optimization for Visible Light Communication System With Lighting Constraints, IEEE Transactions on Signal Processing, 2015, Volume: 63, Issue: 16 , Page s: 4245 - 4256

[31] Gábor, Fekete; Gergely, Mészáros; Eszter, Udvary; Gábor, Fehér; Tibor, Berceli: Visible light communication channel disturbances and examination of the modulation formats, International Journal of Microwave and Wireless Technologies 8: 8 pp. 1163-1171. Paper: MRF1500, 9 p. (2016)

[32] Harald Haas: LiFi: Conceptions, misconceptions, and opportunities, 2016 IEEE Photonics Conference (IPC), Page s: 680 - 681

[33] www.lificonsortium.org

[34] Cheng Chen; Rui Bian; Harald Haas: Omnidirectional Transmitter and Receiver Design for Wireless Infrared Uplink Transmission in LiFi, 2018 IEEE International Conference on Communications Workshops

[35] Aimin Tang; Chao Xu; Bangzhao Zhai; Xudong Wang: Design and Implementation of an Integrated Visible Light Communication and WiFi System, 2018 IEEE 15th International Conference on Mobile Ad Hoc and Sensor Systems

[36] Cheong, Y.-K.; Ng, X.W.; Chung, W.-Y., "Hazardless Biomedical Sensing Data Transmission Using VLC," Sensors Journal, IEEE, vol.13, no.9, pp.3347,3348, Sept. 2013.

[37] Hyun-Seung Kim; Deok-Rae Kim; Se-Hoon Yang; Yong-Hwan Son; Sang-Kook Han, "An Indoor Visible Light Communication Positioning System Using an RF Carrier Allocation Technique," Journal of Lightwave Technology, vol.31, no.1, pp.134,144, Jan.1, 2013.

[38] Optical Wireless Communications, An Emerging Technology, chapter 28: Gabor Feher and Eszter Udvary, VLC Based Indoor Localization, ISBN 978-3-319-30201-0, Springer International Publishing, 2016,

[39] Minchul Kim; Taeweon Suh: A Low-Cost Surveillance and Information System for Museum Using Visible Light Communication IEEE Sensors Journal 2019, Volume: 19, Issue: 4 Page s: 1533 - 1541

[40] Renata Maria Mare; Claudio Luiz Marte; Carlos Eduardo Cugnasca: Visible Light Communication Applied to Intelligent Transport Systems: an Overview, IEEE Latin America Transactions, 2016, Volume: 14, Issue: 7, Page s: 3199 - 3207

[41] Mohammed Elamassie; Farshad Miramirkhani; Murat Uysal: Performance Characterization of Underwater Visible Light Communication, IEEE Transactions on Communications, 2019, Volume: 67, Issue: 1, Pages: $543-552$

[42] Tamás, Szili; Balázs, Matolcsy; Gábor, Fekete: Water Pollution Investigations by Underwater Visible Light Communications Paper: Tu.P.14, 17th International Conference on Transparent Optical Networks, ICTON 2015

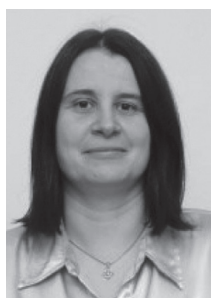

Eszter Udvary (M'98) received the Ph.D. degree in electrical engineering from Budapest University of Technology and Economics (BME), Budapest, Hungary, in 2009. She is currently an Associate Professor at BME, Department of Broadband Infocommunications and Electromagnetic Theory, where she leads the Optical and Microwave Telecommunication Lab. Dr. Udvary's research interests are in the broad areas of optical communications, include optical and microwave communication systems, Radio over fiber systems, optical and microwave interactions and applications of special electrooptical devices. 